\title{
Application of Advanced CFD Tools for High Reynolds Number Testing
}

\author{
S. Melber-Wilkending and G. Wichmann \\ German Aerospace Center, DLR, Institute of Aerodynamics and Flow Technology, \\ Braunschweig, Germany
}

\begin{abstract}
Numerical simulations of the DLR F11 high lift half model in landing configuration in the test section of the ETW wind tunnel have been carried out with the unstructured CFD code DLR TAU. The numerical results are compared to measurements of the cryogenic wind tunnel ETW performed within the EU project FLIRET. The tests have been conducted with three different peniche heights of the F11 model to determine the influence of the so called half model mounting effects on the aerodynamic characteristics of model flow. Based on the numerical simulation of the half model tests the peniche effects and the wind tunnel flow itself are analyzed. The capability of the used CFD code for high Reynolds number testing is demonstrated.
\end{abstract}

\section{Introduction}

$\mathrm{D}$ uring the last years advanced modern procedures for CFD flow simulation have been further developed in a great extent. Amongst others they are able to support the wind tunnel experiment in the sense that their solutions can answer questions related to all problems of wind tunnel interference effects for high Reynolds number testing, too. In particular using unstructured codes for the flow simulation around complex configurations also complete wind tunnel flows can be handled with the required accuracy and justified effort. Thus the critical examination of existing wind tunnel correction procedures and their improvement is made possible, leading to more reliable procedures for the prediction and extrapolation of the wind tunnel experiment to free flight. Within the DLR project ForMEx [1-5] the numerical simulation and respectively the analysis of the wind tunnel experiment considering all geometrical and aerodynamic conditions has been performed in order to improve the wind tunnel testing technique for low speed tunnels. In the process also model deformation effects have been considered using flow/structure coupling methods. From the deviations detected by careful comparisons of the experimental data with the results of the numerical simulation of the experiment two main statements can be derived: On the one hand they help to identify the limits of existing wind tunnel correction methods and possibly lead to certain improvements; on the other hand they also serve for validation and improvement of numerical methods. Thus based on the ForMEx project the activities within the European project FLIRET demonstrate the CFD potential to support high Reynolds number testing in the ETW.

This paper presents selected results achieved during the FLIRET project work, task 3.2 titled "Half-model mounting effects on flow characteristics". They are based on numerical simulations using the hybrid unstructured code of DLR TAU compared with the ETW measurements. As test configuration the high lift half model DLR F11 in landing condition has been used. The numerical treatment of the wind tunnel flow is discussed. Comparisons of the numerical and the experimental results are presented indicating the Reynolds influence on the aerodynamic coefficients of the DLR F11 high lift configuration at variable peniche heights. The numerical results concerning the peniche influence and the wind tunnel interference show that CFD has the potential to improve the wind tunnel technique also for high Reynolds numbers.

The presented results will lead to the statement that a consequent further development of the advanced CFD tools is a promising way for better wind correction methods as well as for more accurate free flight predictions.

\section{Flow Solver}

For the solution of the RANS-equations a three-dimensional finite volume code - the DLR TAU-code - is used. The DLR TAU-code is a hybrid unstructured flow solver based on a three-dimensional finite volume scheme. The governing equations are solved on a dual background grid, which, together with the edge-based data structure, allows running the code on any type of cells. The solver is part of the MEGAFLOW-software package [6]. The temporal gradients are discretized using a multi-step Runge-Kutta scheme. The inviscid fluxes are calculated employing a central method with scalar dissipation. The viscous fluxes are discretized using central differences. In order to accelerate the convergence to steady state, local-time stepping and a multigrid technique based on 
agglomeration of the dual-grid volumes are employed. Because of the low free-stream Mach number preconditioning was used to improve accuracy and to enhance the convergence to steady state [7].

For the closure of the Reynolds-averaged equations the k $\omega$-SST turbulence model of Menter [8] is used, which combines robustness with the applicability for partly detached flows. Finally, the central JST-scheme in combination with $80 \%$ matrix dissipation ensures numerical flow solutions with low numerical dissipation. To increase the convergence, an implicit time-integration (LU-SGS) was [9] is implemented in the TAU code. All simulations have been done including laminar/turbulent transition based on an automated transition prediction.

In order to simulate a numerical wind tunnel special boundary conditions are needed because preventing from simulating the complete wind tunnel. Therefore, the settling chamber, the nozzle, the test section with closed slots and the re-entry section including the sword for full model tests is treated. Whereas the simulation of the complete wind tunnel is not needed for the simulation of the mentioned parts a correct simulation of the growing and development of the boundary layer on the walls the consideration of the nozzle and therefore the test section is necessary. There, the half model is mounted on the ceiling and therefore partly in the boundary layer of the wall. Details of the numerical simulation of a wind tunnel can be found in [1-5].

\section{Configuration \& Grid Generation}

The DLR F11 configuration is based on a typical transport aircraft configuration. The high-lift configuration used here is the landing configuration of this aircraft with the slat deployed at 26.5 degree and flap deployed on 32.0 degree. The geometrical sizes of this configuration are a reference area of $0.419 \mathrm{~m}^{2}$ and reference length of $0.347 \mathrm{~m}$. The wing and high-lift elements of the wind tunnel model in half-model configuration are made from a special steel for cryogenic testing, the fuselage has a carbon fiber structure. In Figure 1 the configuration is shown. For the halfmodel peniches with three different heights are available: $45 \mathrm{~mm}, 72 \mathrm{~mm}$ and $100 \mathrm{~mm}$ (existing standard peniche).
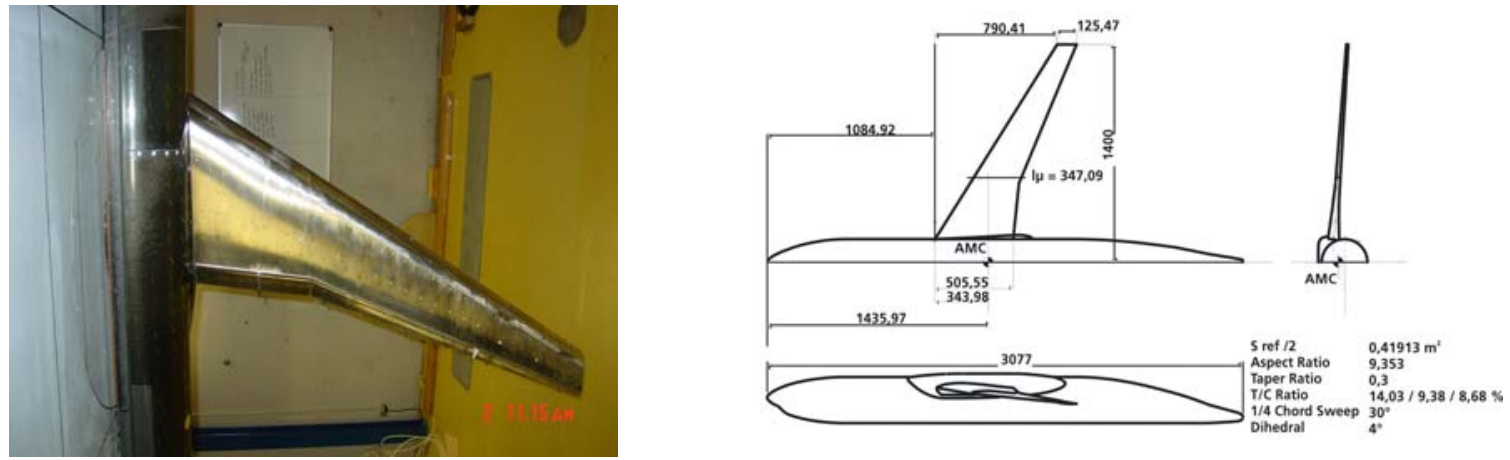

Figure 1: DLR F11 high-lift configuration, wind tunnel model and main dimensions

The numerically simulated wind tunnel is the cryogenic wind tunnel ETW in Cologne - compare Figure 2. The free stream conditions at Mach number $\mathrm{Ma}=0.2$ have been defined for a low and high Reynolds number case. For the low Reynolds number the settings are: Pressure $p=148752 \mathrm{~Pa}$, density $\rho=1.743 \mathrm{~kg} / \mathrm{m}^{3}, \operatorname{Re}=2.28 \cdot 10^{6}$. For the high Reynolds number case the settings are: Pressure $p=257870 \mathrm{~Pa}$, density $\rho=7.903 \mathrm{~kg} / \mathrm{m}^{3}$ and $\mathrm{Re}=14.9 \cdot 10^{6}$.

The grids dedicated for the hybrid unstructured DLR TAU code have been built with the grid generator CENTAUR from CentaurSoft. It is a hybrid grid generator for complex configurations, which is split up in three stages: surface, prism or hexahedra (boundary layer) and tetrahedral generator. The resulting grids for the F11 highlift configuration including the wind tunnel have about 15 million grid points.

To be able to simulate numerically the changes of angle of attack of the wind tunnel model without regenerating the grids for each angle of attack the chimera technique has been used. In Figure 3 the mesh of the test section including the chimera boundaries is shown. By rotating both meshes against each other (blue and red one) the angle of attack of the wind tunnel model can be changed. The advantages of this technique are:

- No regeneration of grids for different angle of attack, which saves time and computer power

- Constant grid quality for all angles of attack

- Prevention of list hysteresis (numerical and physical one)

- Fully automatic numerical simulation of lift polars without any invention of the user. 

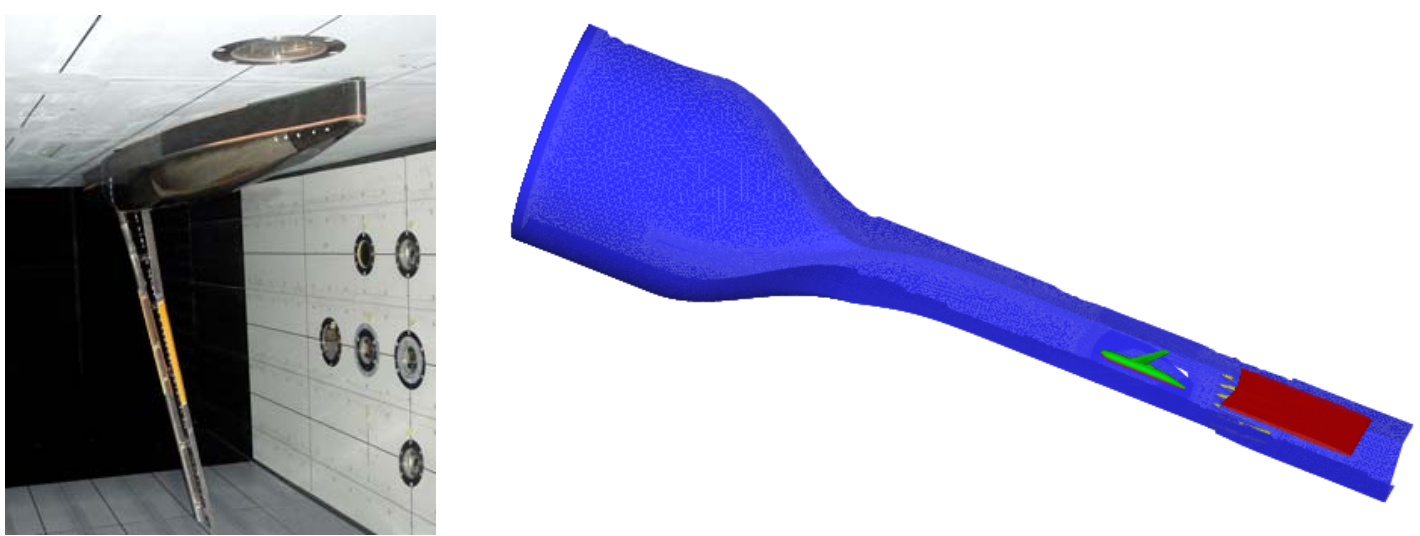

Figure 2: DLR F11 model in ETW test section and the numerical simulated domain with ETW settling chamber, nozzle, test section with F11 high-lift configuration, re-entry section.
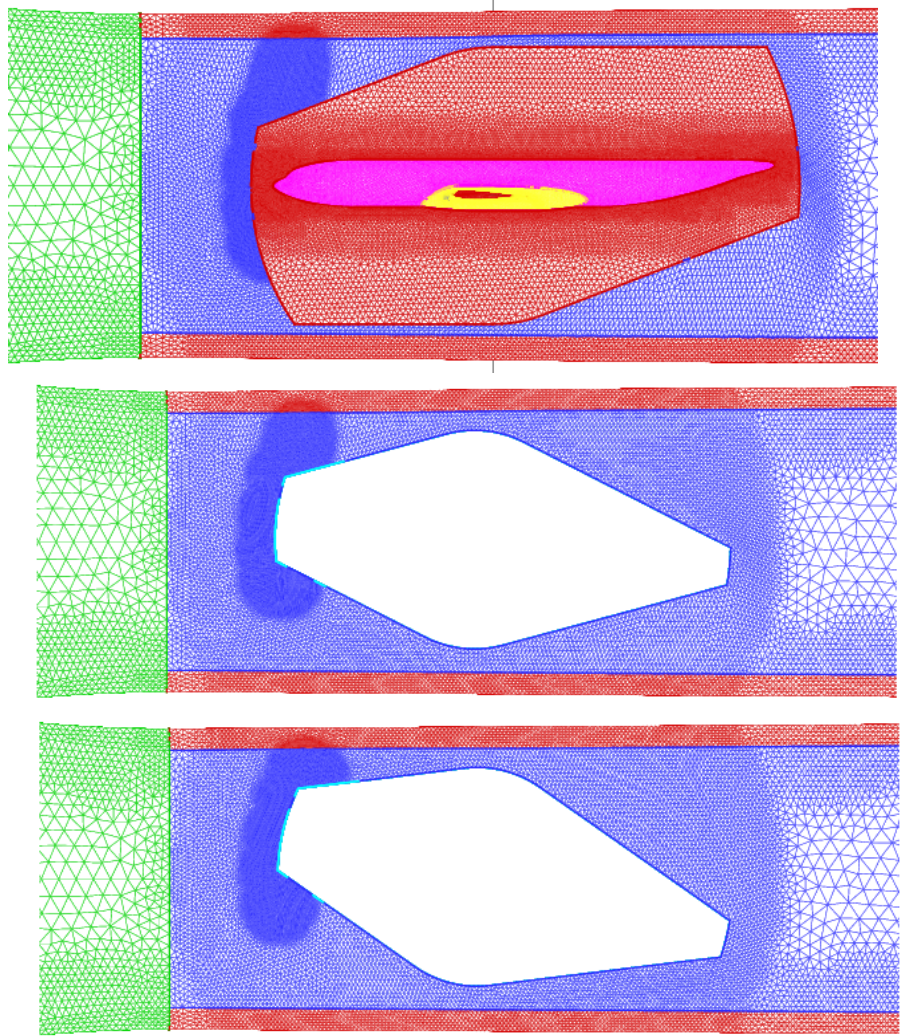

Figure 3: Mesh of test section, F11 high-lift configuration and chimera boundaries (top) and deformed background mesh (middle \& bottom)

A problem occurs using chimera technique in combination with the F11 configuration and the ETW wind tunnel. The length of the fuselage is greater then the height of the test section, compare Figure 3. This means in turn, the chimera boundaries have only a small range of angles of attack, in which both grids can be rotated inside each other before the fuselage overlaps the grid of the test section chimera boundaries. This leads to a range of angles of attack of about 10 degrees, which is much lower then the necessary 25 degrees.

The solution of this problem is a combination of chimera technique and grid deformation. The change of the angle of attack is done using chimera. If the fuselage touches the chimera boundaries (boundaries of the test section grid) they are moved using grid deformation. In Figure 3 two different positions of the chimera boundaries are shown. Looking on the elements of the mesh only a small deformation of the cells itself can be found, which means that a very good grid quality is still available. 


\section{Results}

\section{A. Reynolds-Number Influence}

In this section some selected results are presented concerning the Reynolds number influence on the aerodynamic lift and drag coefficients of the DLR F11 high lift half model in landing configuration at different peniche heights. The data have been achieved during the FLIRET test campaign in ETW.

Three peniche configurations of the DLR F11 model have been tested with heights of $100 \mathrm{~mm}, 72 \mathrm{~mm}$ and 45 $\mathrm{mm}$ at a Reynolds number range of $\mathrm{Re}=2.3$ to $25.0 \times 10^{6}$. The tests have been performed at $\mathrm{M}=0.2$, the evaluated results represent the corrected test data. The pictures in Figure 4 show the model with the $100 \mathrm{~mm}$ peniche.
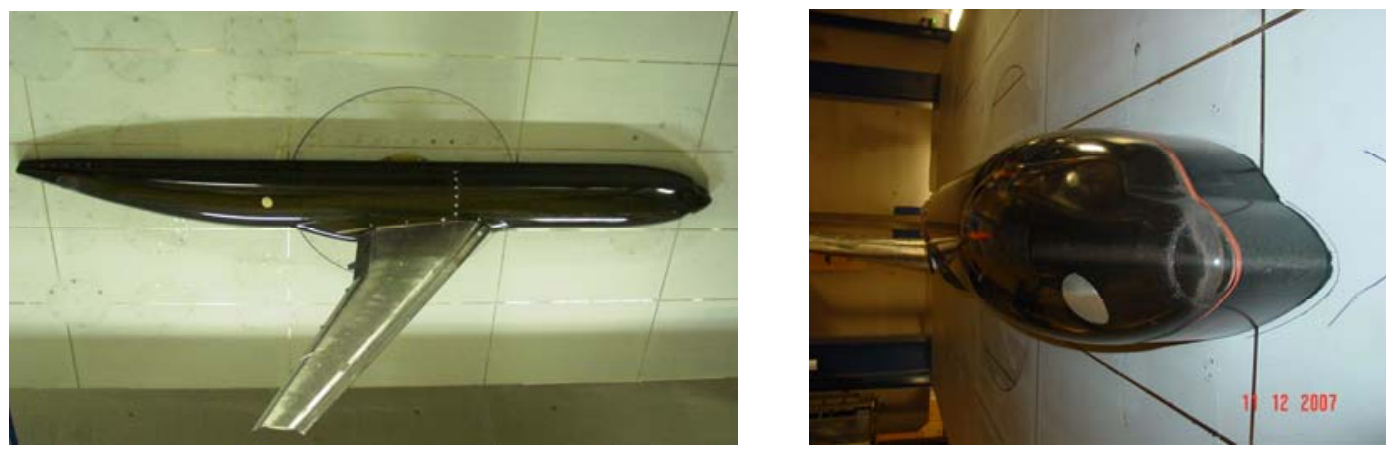

Figure 4: DLR F11 high lift model in ETW with $100 \mathrm{~mm}$ peniche

Figure 5 shows the measured lift curve as well as the drag polar for one selected peniche height at different Reynolds numbers. As the curve characteristics in dependence of Reynolds number are similar for all peniche heights investigated the figure only shows the case of the $100 \mathrm{~mm}$ peniche exemplary:

- The lift curves show the lowest values at the lowest Reynolds number, i.e. $\operatorname{Re}=2.310^{6}$. This also holds for the $\mathrm{C}_{\mathrm{L} \text { max }}$. The differences between the curves of the remaining Reynolds numbers turn out to be not significant except in the $\mathrm{C}_{\mathrm{L} \text { max }}$ region were the highest values are reached for the case with the highest Reynolds number $\mathrm{Re}=25 \times 10^{6}$.

- $\quad$ The drag polars have their highest values at lower Reynolds numbers.
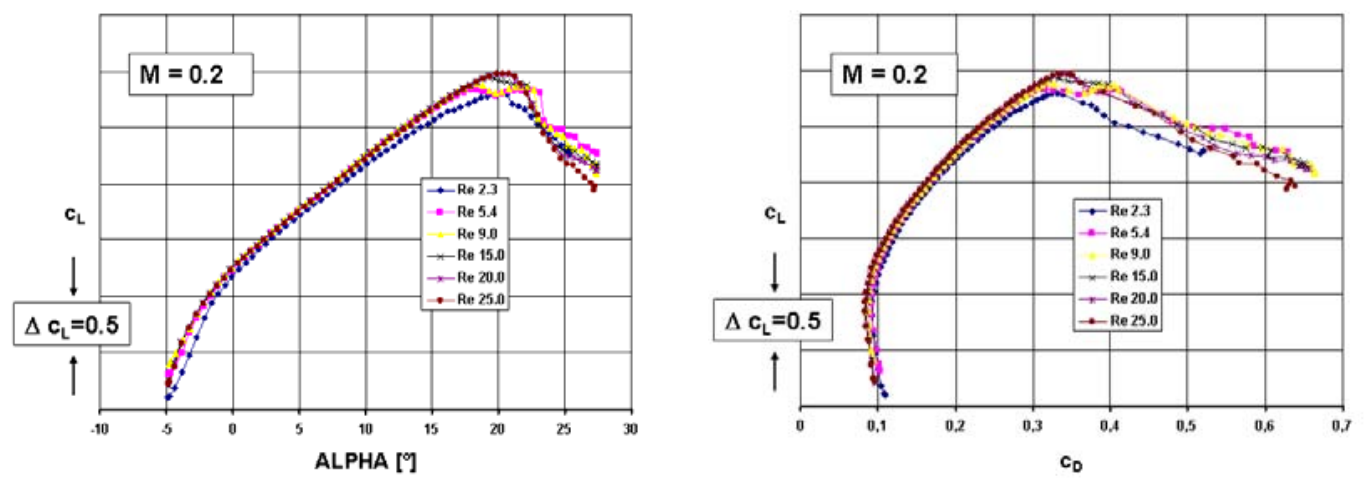

Figure 5: Reynolds number influence on lift curve and drag polar at peniche height $100 \mathrm{~mm}$

Figure 6 presents the Reynolds number influence for the three investigated peniche heights of the F11 model for the lift and drag coefficient at the angle of attack $\alpha=15$ degree, linear interpolated from test values.

The evaluated lift coefficients (Figure 6 left) show that due to the aspect ratio and the displacement effect of the peniche configurations the lift values increase with increasing peniche height with the exception of the case at the lowest Reynolds number $\mathrm{Re}=2.3 \times 10^{6}$ where additional transition effects for the lowest peniche height lead to a different behavior. With increasing Reynolds number for all peniche heights the lift coefficient reaches a maximum value, for the $100 \mathrm{~mm}$ peniche at $\mathrm{Re}=15 \times 10^{6}$ and for the $72 \mathrm{~mm}$ and the $45 \mathrm{~mm}$ peniche at $\mathrm{Re}=9 \times 10^{6}$. In the last case the lift value nearly keeps constant with further increasing Reynolds number, whereas for the higher peniche heights a decrease of $\mathrm{c}_{\mathrm{L}}$ is stated with further increasing Reynolds number. 

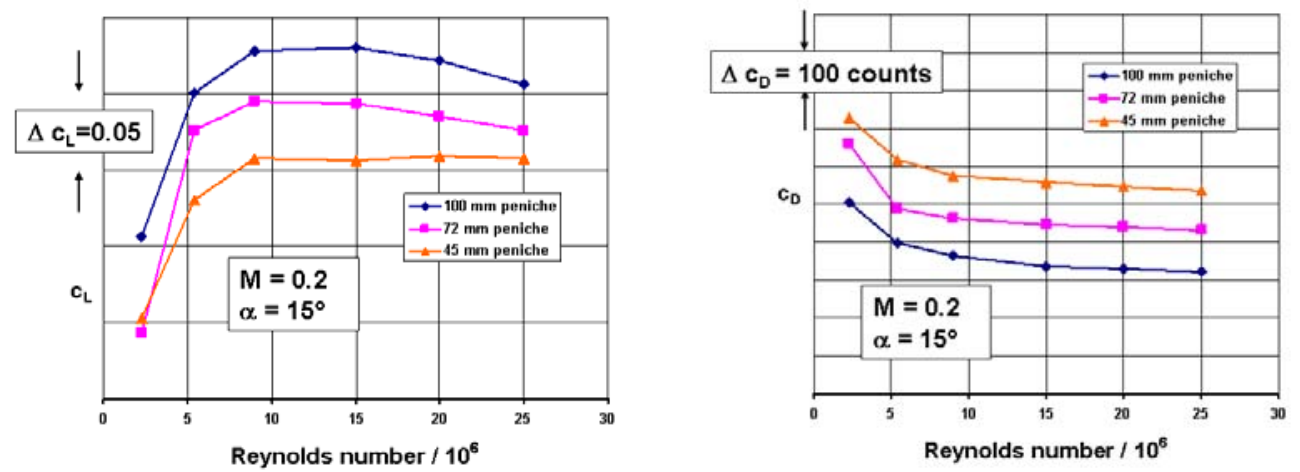

Figure 6: Reynolds number effect on lift and drag coefficient at different peniche heights at $\mathrm{M}=\mathbf{0 . 2}$ and $\alpha=15^{\circ}$

The corresponding plot of the evaluated drag coefficients (Figure 6 right) shows a clear Reynolds number dependency of the drag values for the three peniche heights. The drag coefficient decreases with increasing Reynolds number significantly up to $\operatorname{Re}=9.0 \times 10^{6}$, less strongly when the Reynolds number further increases. Mainly due to the aspect ratio effect of the different peniche configurations on the induced drag coefficient the lowest values are measured for the highest peniche height and the highest values for the lowest peniche height.

The Reynolds number influence on the maximum lift behavior is presented in Figure 7. It can be stated:

- With increasing peniche height the displacement effect of the peniche also increases leading to higher $\mathrm{C}_{\mathrm{Lmax}}$-values. Therefore the lowest $\mathrm{C}_{\mathrm{L} \text { max }}$ can be found for the lowest peniche height and the highest for the highest peniche.

- $\quad$ The maximum lift coefficient is clearly increasing with Reynolds number with a stronger descent at lower Reynolds numbers.

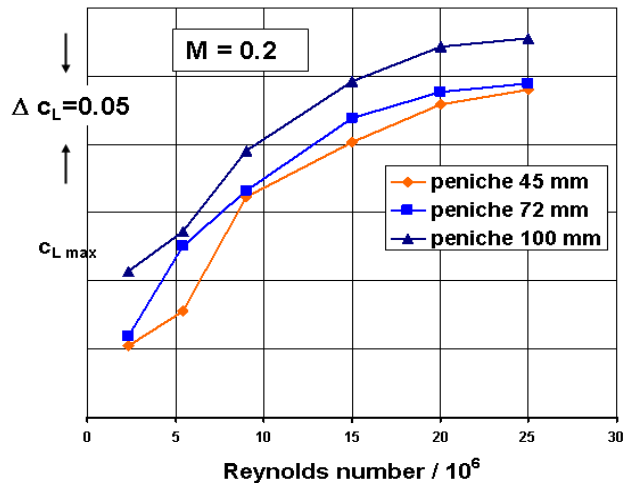

Figure 7: Reynolds number effect on maximum lift coefficient at $\mathrm{M}=\mathbf{0 . 2}$

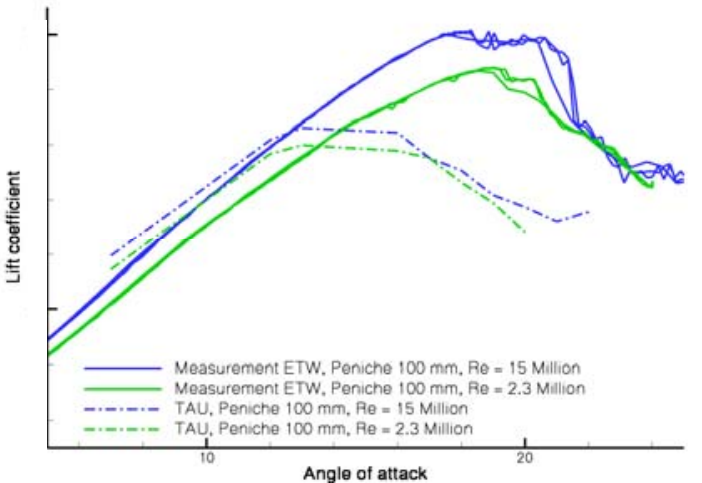

Figure 8: Comparison ETW test - CFD at $\mathrm{M}=0.2$

In Figure 8 the Reynolds number influence for the case with the $100 \mathrm{~mm}$ peniche is shown for the Reynolds numbers of 15 and 2.3 million. First, comparing both numerical simulations (dash-dotted lines) two typical effects can be found. With increasing Reynolds number the boundary layer gets thinner. This leads to an additional cambering of the airfoils, which results in an increased lift. The second and main effect due to the Reynolds number results from the effect in the gap areas: Due to the thinner boundary layers, the available gap increases and therefore the mass flow in this region. This increases the circulation and leads to an additional lift, which can be found in Figure 8.

Regarding the measurements in the ETW wind tunnel (see also Figure 8) the same effects as for the numerical results can be found. However, in the measurements the effect of model deformation takes place, which is absent in the numerical simulations. This fact can be used to separate the deformation from the other aerodynamic effects: The difference between both numerical simulations combines all effects due to the change of the Reynolds number 
without the deformation. Comparing this difference with the difference between both measurements, the difference between both "differences" represents the effect of model deformation.

Analyzing again Figure 8, a nearly constant difference for the numerical simulations can be found, whereas the difference between both measurements shows an increasing effect with increasing angle of attack. This clearly shows the character of model deformation: With increasing angle of attack the aerodynamic load on the model increases and therefore the deformation although increases with a resulting change in aerodynamic results.

This effect although puts a new light on the comparison of measurements and numerical simulation. With increasing Reynolds number the dynamic pressure increases about 55\%. The dynamic pressure is the main driver for the resulting deformation. Comparing now the measurements and numerical simulation for the lower Reynolds number, the curves are parallel, whereas for the higher Reynolds number the measurement shows an increased gradient compared to the numerical simulations, a clear indication of the deformation.
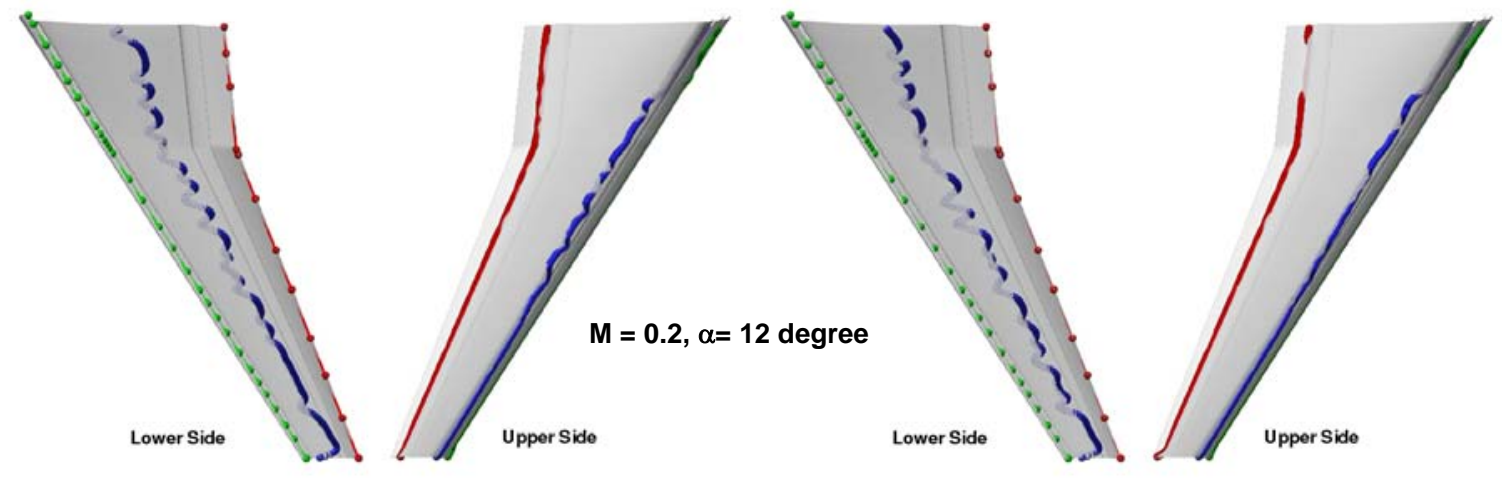

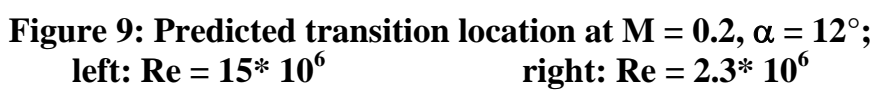

In Figure 9 the location of the predicted transition position is shown, at left hand side at $\operatorname{Re}=15^{*} 10^{6}$, on right hand side at $\operatorname{Re}=2.3^{*} 10^{6}$. No significant change induced by the change of the Reynolds number can be found. The main reason is probably the neglect of the cross flow, which can have a significant amount at Reynolds number of 15 million, whereas the Tollmien-Schlichting driven transition location is constant.

\section{B. Peniche \& Wind-tunnel Influence}

In this section the half model test technique and with it the so called "peniche" which is used in the most cases of half model testing will be discussed by its aerodynamic effect on the model flow. Further on, the influence of the wind tunnel itself with its walls will be considered.

\section{Half-model Test Technique (peniche effect)}

To get the influence of the peniche its height will be varied in two steps. In the first step the peniche will be completely removed, the symmetry plane of the fuselage is therefore direct on the tunnel floor and with it the fuselage is partly located in the boundary layer of the wind tunnel wall. For comparison reasons the configuration with the peniche of $100 \mathrm{~mm}$ is used.

In order to visualize the differences between the configurations considered so called "difference pictures" are used, as shown in Figure 10 and 11. In these pictures for the angle of attack, the longitudinal velocity and the crosswise velocity the differences between two configurations in the flow field are shown and therefore the value from the first subtracted from the value from the second configuration. This kind of pictures enables the visualization of small differences between two configurations, which often cannot be detected comparing both flow fields directly. 

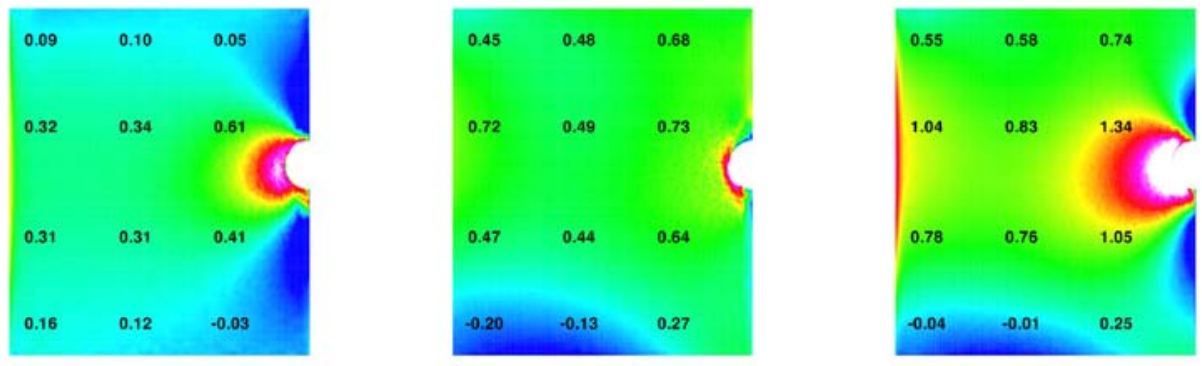

angle of attack

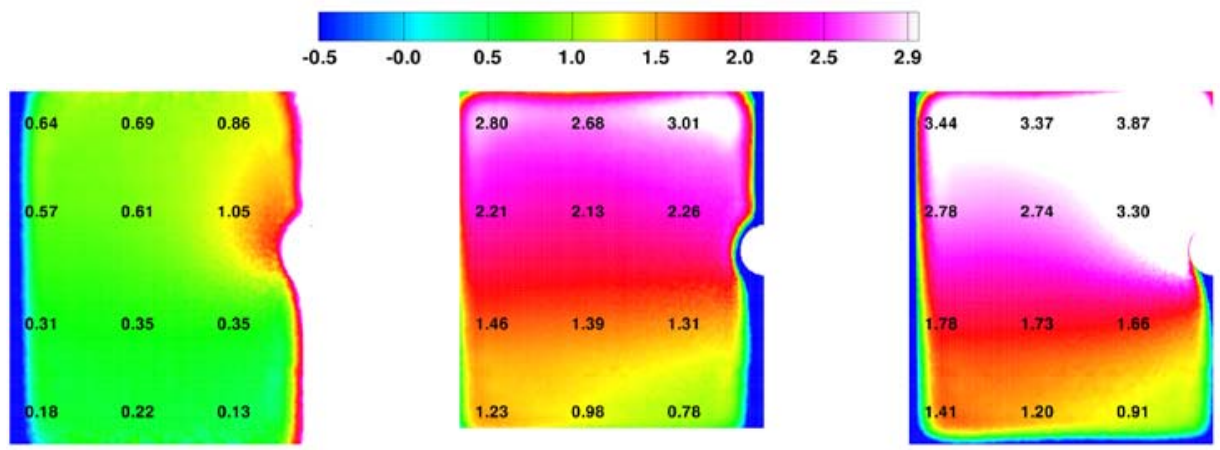

velocity in free stream direction

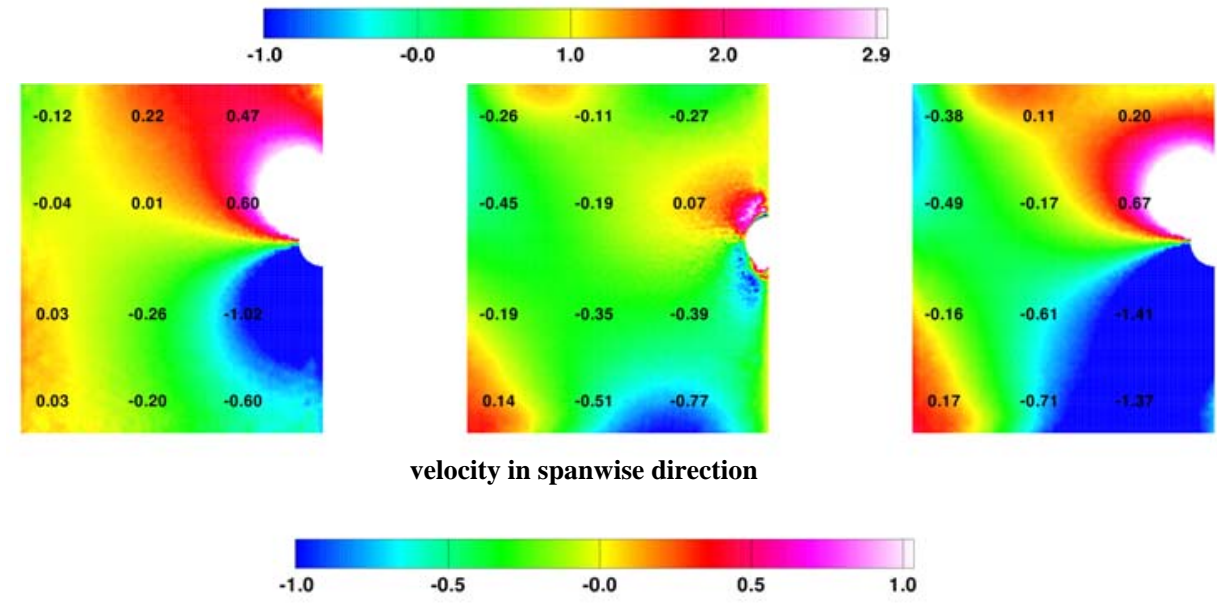

Figure 10: Difference pictures between configuration with peniche $(100 \mathrm{~mm})$ and without peniche (left), without peniche and free flight (middle) and with peniche $(100 \mathrm{~mm})$ and free flight (right). Cut at $x=-0.5$ (front of the wing) through the flow field, angle of attack 12 degree; shown value: angle of attack (top), velocity component in free stream direction (middle), velocity component in span wise direction (bottom)

For both configurations with and without peniche an additional angle of attack on the inboard wing can be found of about 1 degree in case of a peniche, Figure 10. On the outboard wing the angle of attack is nearly unchanged. In the side view in a cut near to the wing root through the fuselage clearly the local influence of the peniche on the fuselage can be found - Figure 11. The velocity of the cross flow is reduced above und increased below the fuselage, both in size of $0.5 \mathrm{~m} / \mathrm{s}$. This influence is again only on the inboard wing (Figure 10). In flow direction on upper side of the inboard wing an acceleration of the flow of about $0.5 \mathrm{~m} / \mathrm{s}$ with peniche (Figure 10), in front of the configuration a deceleration can be found (Figure 11). 

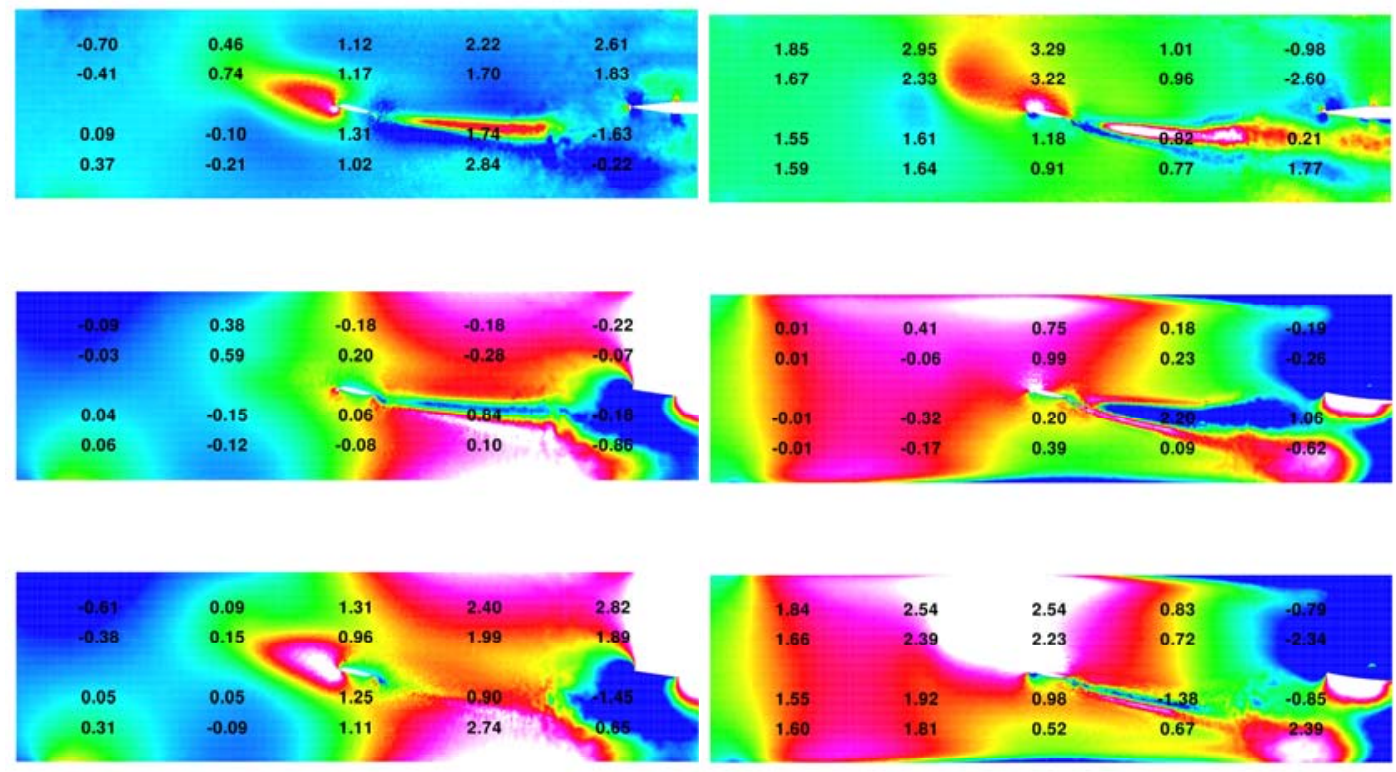

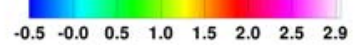

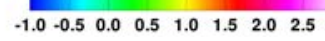

Figure 11: Difference pictures between configuration with peniche $(100 \mathrm{~mm})$ and without peniche (top), without peniche and free flight (middle) and with peniche $(100 \mathrm{~mm})$ and free flight (bottom). Cut at $y=0.72$ (inboard wing) through the flow field in span wise direction, angle of attack 12 degree; shown value: angle of attack (left) and velocity component in free stream direction (right)

The reason of these effects is the peniche displacement effect. In case of a fuselage with peniche with zero incidence ( $\alpha=0$ degree) this combination is an additional obstruction for the flow, which leads to an increased flow velocity in the proximity of fuselage. With increasing angle of attack besides the cross sectional area itself a part of the side surface is added, which leads to an induced velocity. Because of the increased flow velocity in cross direction of the fuselage axis the additional local angle of attack is increased. The displacement effect of the peniche decays in span wise direction, because the additional displacement is only present in the area of the fuselage.

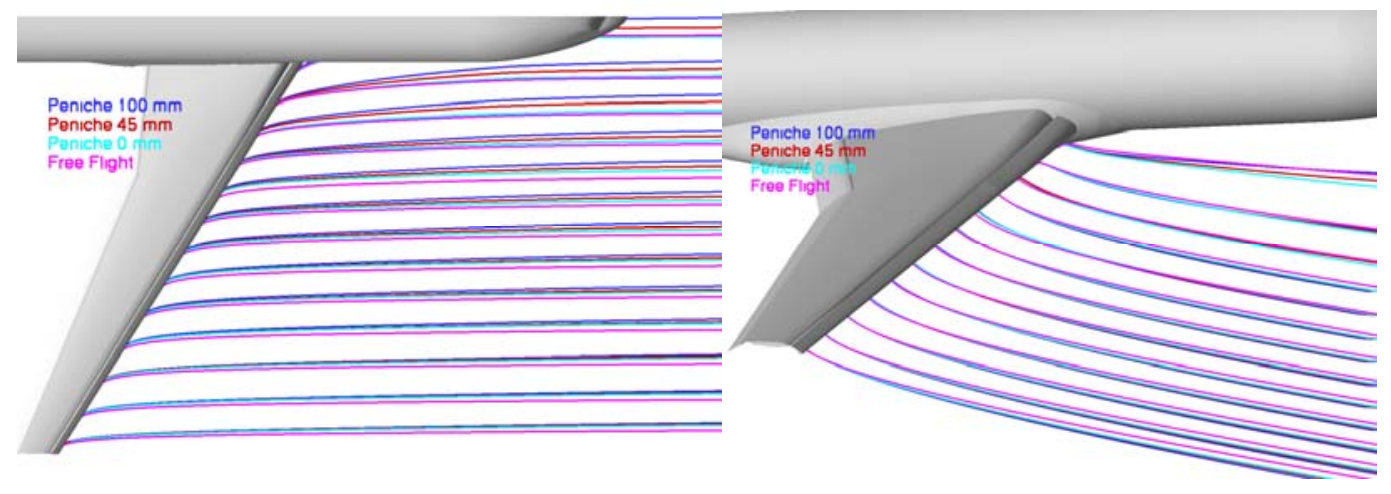

Figure 12: Stagnation point streamlines for different peniche heights - view from above, angle of attack 12 degree

For clarification of the peniche displacement effect in Figure 12 the stagnation points on the slat and the associated stream lines on different span wise positions are shown. The side view shows the already discussed increased angle of attack on the inboard section and the decay with increasing span wise positions. Further on the displacement effect of the peniche moves the stagnation point stream lines more in span wise direction, whereas this effect is reduced with increasing span wise position. Because the stream lines are backtracked starting from the 
stagnation point, in the case with peniche they are emanating from a more inboard area compared to a case without peniche.

In the same way streamlines on the surfaces (Figure 13) are moved in span wise direction because of the displacement effect. This additional cross sectional flow has its strongest value at the inboard section on the trailing edge of the wing in the area of the inboard flap, because the flow is already decelerated in this area, therefore the peniche influence higher influence on the flow direction.
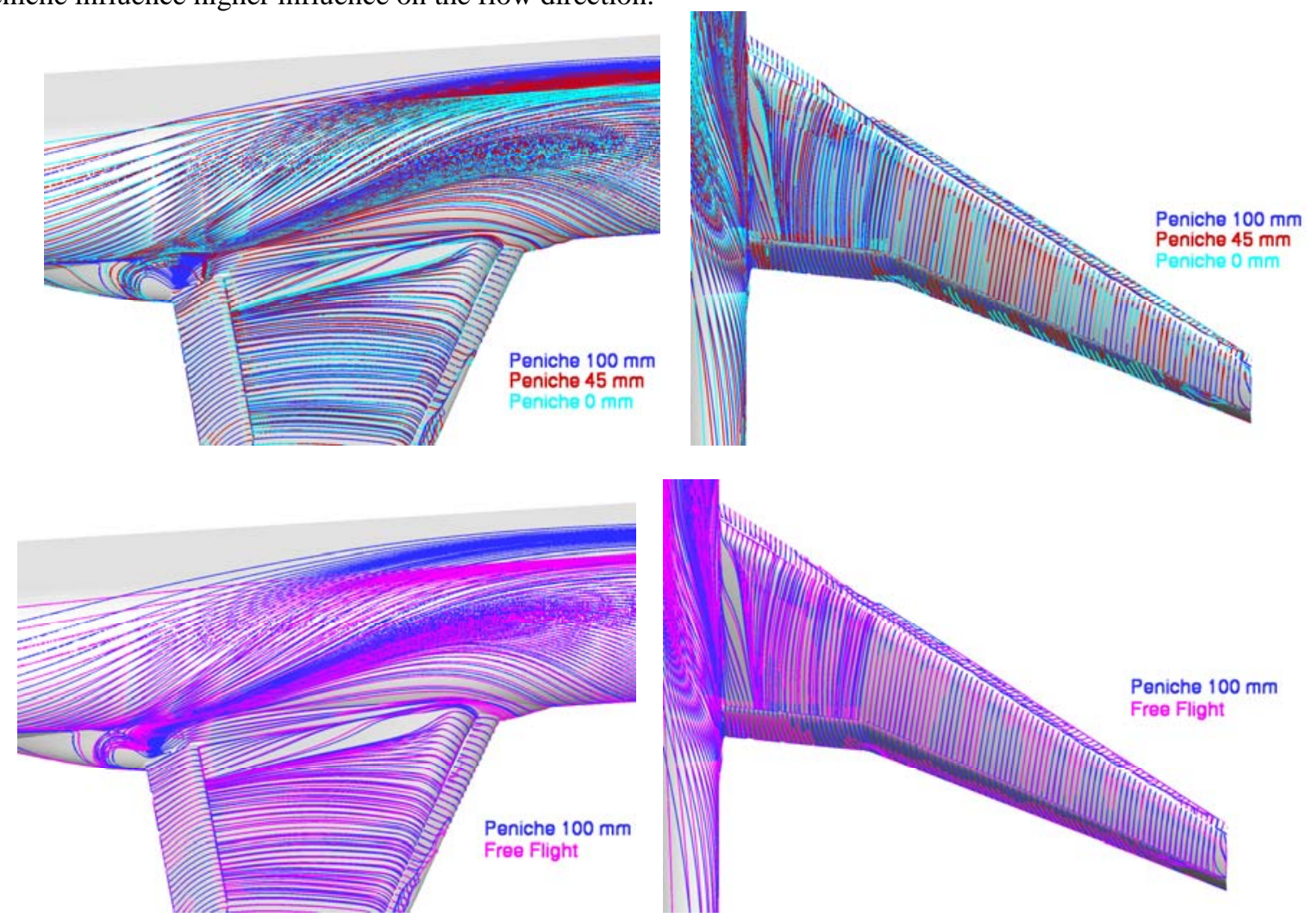

Figure 13: Surface streamlines for different peniche heights, angle of attack 12 degree

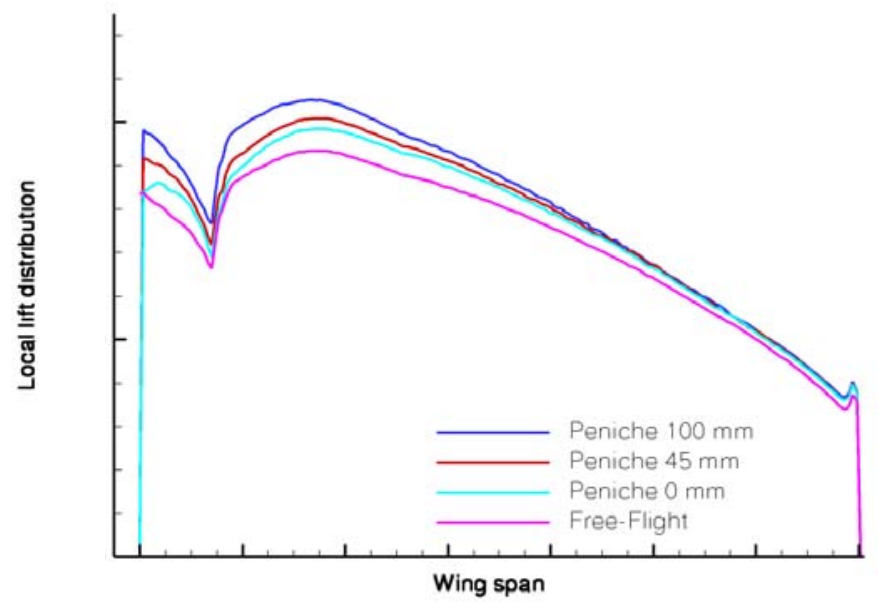

Figure 14: Span wise lift distribution for different peniche heights and free flight, angle of attack 12 degree 
In Figure 14 the lift distribution along the wing span for three different peniche heights and at free flight is shown. Clearly the change of the flow in span wise direction between wind tunnel and free flight and mainly the influence of the peniche on the inboard flow can be found. Further on, with any peniche a difference to the free flight results is clearly visible.

Overall the peniche and its displacement effect leads to an additional flow velocity on the fuselage surface and the inboard wing compared with a configuration without peniche. Further on the local angle of attack is increased in the inboard wing. The strength of the peniche displacement effect is directly linked with the angle of attack of the configuration, which can be found in the lift polars (Figure 15) by means of an increasing lift slope with increasing peniche height and increasing angle of attack. The peniche influence can be found at each angle of attack, because also in the case without angle of attack an additional displacement takes place due to the peniche.

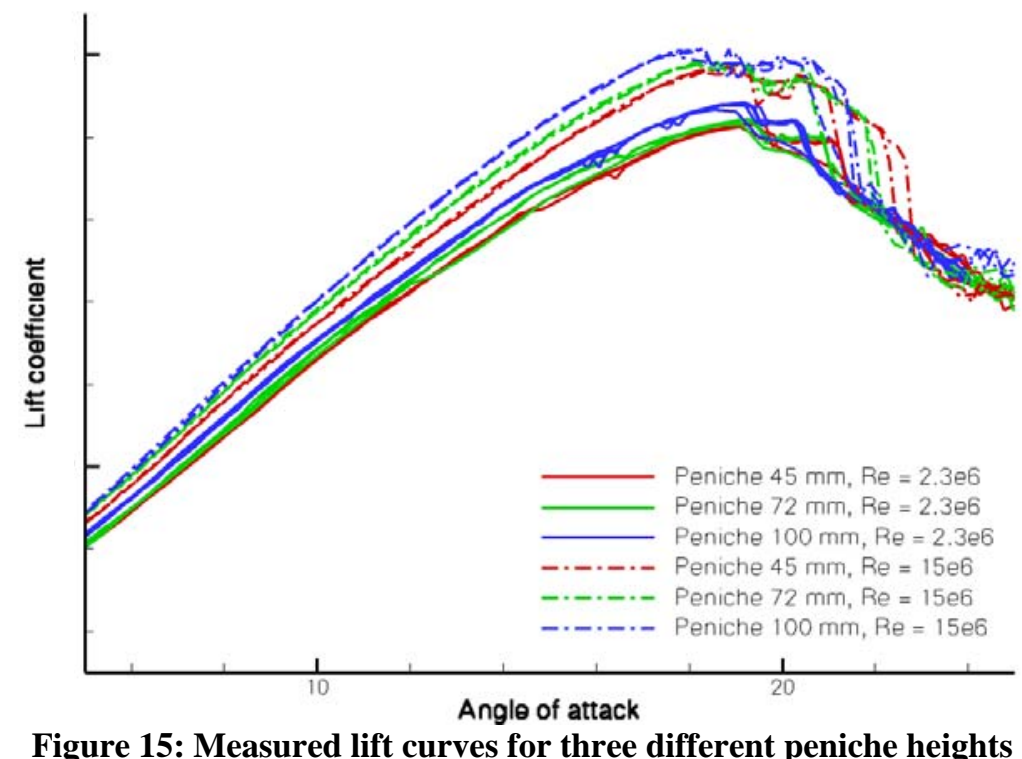

With a variation of the peniche height therefore only the strength of the displacement effect can be changed, but it cannot be avoided in any case. A reduction of the displacement effect is caused by the boundary layer of the wind tunnel wall for an half model test, whereas only a small influence of the angle of attack can be found. To reach an minimal disturbance of the flow for lower angles of attack a peniche with more height is advantageous, at higher angles of attack a smaller one. In principle for each flow condition and therewith angle of attack a peniche height independent of its geometry can be found, which has globally the same displacement as the boundary layer of the fuselage reduced by the boundary layer of the tunnel wall. However the thickness of neither the wall boundary layer nor the displacement along the model is constant and therefore the peniche disturbs the flow in any case.

Thus the "Numerical Wind Tunnel" - the technique simulating such flows with CFD codes - plays an important role for the portability of half model results in the wind tunnel to free flight, because using this numerical wind tunnel a detailed conclusion of the peniche influence on the flow and therewith the change of the characteristics of a configuration can be given.

\section{Wind-tunnel Interference}

In this section the influence of the test section on the flow of the F11 high lift configuration will be shown. Therefore a comparison of the full configuration in free flight with a half model in the wind tunnel without a peniche and the influence of the wall boundary layer are needed. However the second configuration described above can not exist because of the physical boundary conditions, accordingly the wind tunnel interference will be analyzed using two pairs of configurations. The first pair consists of a half model in the wind tunnel without peniche and a full model in free flight. This pair has no peniche interference, but an influence of the boundary layer of the wind tunnel. A significant reduction of the boundary layer interference but with the peniche interference discussed can be reached by the second pair of configurations, i.e. a half model in the wind tunnel on a peniche compared with the free flight. This second pair is furthermore the comparison between wind tunnel and free flight and therefore the basis of a free flight extrapolation of wind tunnel results. 
Again difference pictures are used - here for the determination of the wind tunnel interference. From Figure 8 an additional angle of attack of 1 degree over the complete wing span could be found. The increased angle of attack because of the peniche influence of about 1 degree on the inboard wing is respectively superposed to the wind tunnel influence. Above and below the fuselage a combination of peniche and wind tunnel interference could be found mainly dominated by the movement of the vortices in this area. In the side view (Figure 11) the influence of the wind tunnel can be found in an additional angle of attack of about 0.5 degree in front of the configuration and an additional angle of attack of about 2 degree behind. The wind tunnel interference effect accelerates the flow in free stream direction of about $1.0 \mathrm{~m} / \mathrm{s}$ over the complete wing span (Figure 10), superposed by the same value due to the peniche influence. Below the configuration a deceleration of about $0.2 \mathrm{~m} / \mathrm{s}$ takes place. The cross flow velocity however is unchanged by the wind tunnel interference in contrast to the peniche influence.

The wind tunnel interference can be found further on in the stagnation point streamlines (Figure 12), which show an additional angle of attack over the complete wing span. The influence of the peniche however can only be found on the inboard wing. Because of the additional angle of attack the stagnation line is likewise moved. Looking from above no influence of the wind tunnel can be found.

Comparing the lift curves in Figure 16 one will find the curve of the free flight below the uncorrected curves in the wind tunnel with a slope lower in case of the free flight. The reason is a combined interaction of the wind tunnel and peniche interference leading inside the wind tunnel to an overall increased angle of attack and an increased free stream velocity.

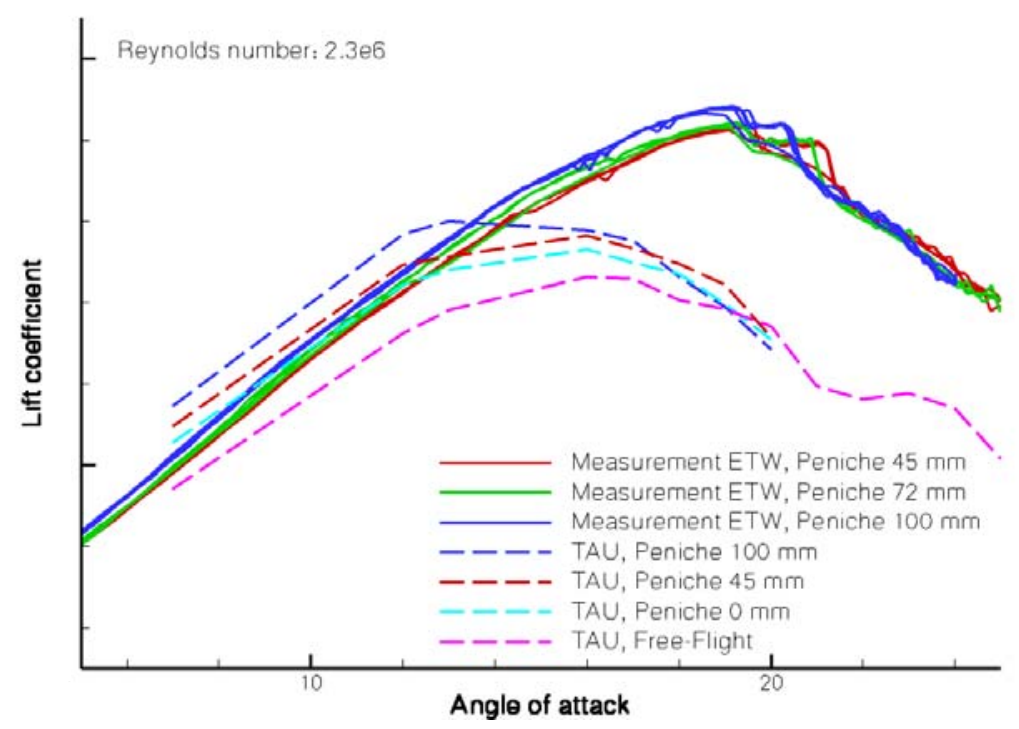

Figure 16: Measured and numerical simulated lift curves

The behavior of the lift curves leads at the first glance to the conclusion that the wind tunnel interference only causes a change in the angle of attack. A detailed check of this postulate by splitting the lift up to the single elements of the aircraft however shows a different distribution in the wind tunnel and free flight for the same lift coefficient. This means in turn a simple correction of the angle of attack is insufficient.

Summarized the peniche and wind tunnel interference can be described as:

- Increased angle of attack and flow speed on the inboard wing (decaying outboard) due to the peniche

- Increased angle of attack and flow speed on the complete wing due to the wind tunnel

- Increased cross flow velocity in the area of the inboard wing due to the peniche

- Increased lift with increased peniche height due to peniche displacement.

\section{Correction of Wind tunnel Interference}

In both sections above the influence of the peniche and wind tunnel on the flow around a high lift configuration has been determined using the so-called "Numerical Wind Tunnel". It can be used for the validation of the current classical wind tunnel corrections at the ETW [10] and therefore as "Numerical Wind Tunnel Correction". Because 
not all correction details had been available in [10], small differences could be found between the correction used at ETW and the one used later when comparing with numerical results (Figure 17).

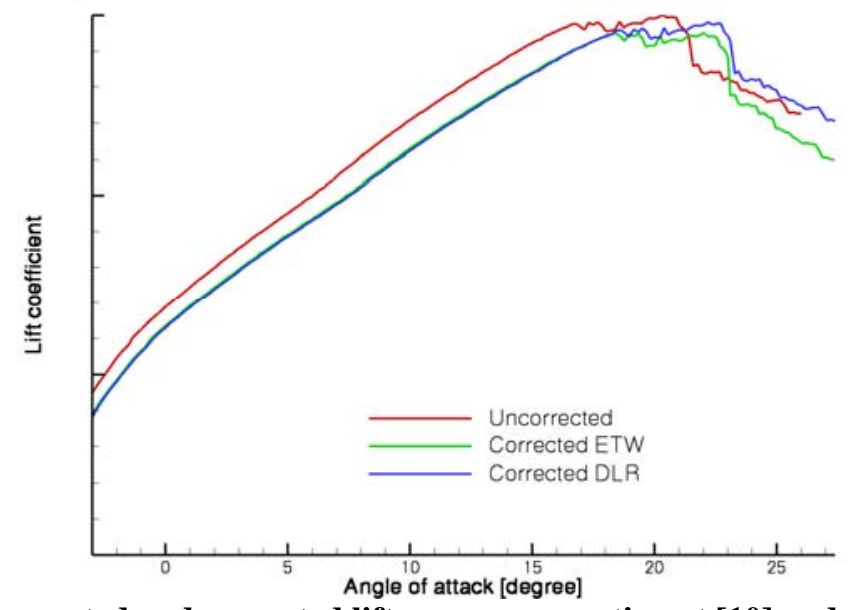

Figure 17: Uncorrected and corrected lift curves, correction at [10] and rebuild by DLR

In Figure 18 the ETW measurements with different peniche heights are shown and compared with the corresponding TAU simulations of the wind tunnel flow as well as the free flight. Overall, a good comparability can be found. Looking into more detail of the differences between the numerical and experimental results, model deformation, bundles of pressure tubes as well as slat and flap tracks itself in the experiment should be kept in mind, because they are not treated in the corresponding numerical approach. Nethertheless, in general a rather good agreement between measurements and unstructured numerical results, including predicted transition locations, has been found.

Overall the calculated lift slope is lower than in the measurement, the simulated maximum lift value is too low as mentioned above. Furthermore, the three measured lift curves for the different peniche heights do not lead to one single curve - although the peniche height is used as a parameter of the classical wind tunnel correction. At this point it is obvious that the classical wind tunnel corrections fail to forecast the influence of the peniche in a correct way, e.g. to predict the free flight.

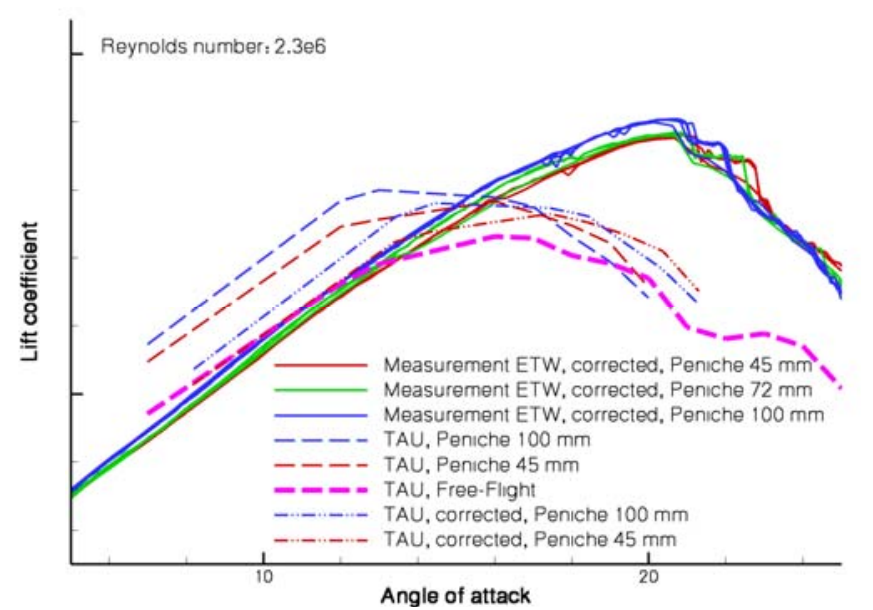

Figure 18: Corrected lift curves with different peniche heights

A reliable validation of the wind tunnel correction cannot achieved with this approach because the differences between measurement and numerical simulation and further on the uncertainty of the wind tunnel corrections are combined inseparably. To evaluate the wind tunnel correction alone a special method is used: The lift curves calculated inside the wind tunnel are corrected with the classical wind tunnel corrections and compared with the free flight simulations. Despite possible differences between numerical flow simulation and flow physics it can be assumed that these differences can be found for the tunnel and free flight simulation consistently and therefore a certain evaluation of the wind tunnel correction can be reached. 
The absence of a modeling of the influence of the wind tunnel boundary layer in the wind tunnel correction is the reason why the corrected lift curves of the configurations with different peniche heights neither coincide among each other nor with the curve of the free flight. Further on a term in the wind tunnel correction is needed to model the increasing displacement effect of the angle of attack for peniche and fuselage. This can be seen in Figure 16 by the increasing distance of the corrected curves with increasing angle of attack. From Figure 16 although the conclusion can be drawn that a peniche lower height then $45 \mathrm{~mm}$ will produce too less lift compared to the free flight because of the influence of the tunnel boundary layer.

Overall the used classic wind tunnel correction can lead, depending on the peniche height, to an good correlation between wind tunnel and free flight, however only because the missing modeling of the boundary layer influence and the replacement of the peniche cancel each other out. Changing the peniche height this compensation fails and significant differences between correction and free flight occur - especially for the local aerodynamic coefficients (e.g. the pressure distribution) this effect can be found.

The potential of the numerical wind tunnel correction for the experimental flow simulation is beside the shown determination of the peniche and wind tunnel influence due to the drop out of any kinds of assumptions also to correct the Reynolds number extrapolation to free flight. Further on, questions which cannot be answered by the classical methods are covered by the numerical wind tunnel correction. Examples are the influence of the mountings, time dependent corrections, powered configurations (propeller influence or thrust determination) or small aspect ratio wings.

The presented results show that only the use of accurate and high resolution CFD can help to solve problems related to wind tunnel correction methods and free flight predictions generating numerical solutions of the complete wind tunnel flow as well as the of the free flight. For the same reason the question dealing with the an optimum peniche for different Reynolds numbers gained from wind tunnel tests cannot be answered satisfactory because any information of the free flight condition is missing.

\section{Conclusion}

The aim of the present paper has been to demonstrate the potential of modern CFD methods for the analyses of complete wind tunnel flows including models up to high Reynolds numbers. Using the example of the ETW wind tunnel flow the determination of the peniche and wind tunnel influences on the flow around the DLR F11 half model high lift configuration could be achieved by the application of the so called "Numerical Wind Tunnel". Hereby the currently used classical wind tunnel corrections at ETW have been validated using this technique and therefore called "Numerical Wind Tunnel Correction". In the following the results of the investigations are concluded:

- Analyzing the Reynolds number influence and the model deformation effect from the measurement data it has been found that the lift curves show the lowest values at the lowest Reynolds number, which also holds for the maximum lift. The differences between the curves of the remaining Reynolds numbers turn out to be not significant except in the $\mathrm{C}_{\mathrm{L} \text { max }}$ region where the highest values are reached for the case with the highest Reynolds number $\operatorname{Re}=$ $25 * 10^{6}$. Further on, the drag coefficients show a clear Reynolds number dependency for the three investigated peniche heights. The drag coefficient decreases significantly with increasing Reynolds number up to $\mathrm{Re}=9.0 \times 10^{6}$ but less strongly when the Reynolds number further increases. With increasing peniche height the lift coefficients also increase whereas the drag values decrease mainly due to an aspect ratio effect induced by the different peniche configurations. The highest values of the maximum lift coefficients have been found for the highest peniche height of $100 \mathrm{~mm}$ followed by the values for the lower peniche heights 72 and $45 \mathrm{~mm}$.

Comparing measurements and CFD it has to keep in mind that of course in the measurements the model deformation effect takes place which is absent in the numerical simulations. Thus the effect of deformation can be separated numerically from the Reynolds number effect. It has been found that with increasing angle of attack the aerodynamic load of the model in-creases and therefore deformation also increases which results in a change of aerodynamic data.

- The influence of the half model testing technique and the usage of a peniche to reduce the influence of the tunnel wall boundary layer has been analyzed in detail. The main peniche effect on the model flow is based on its additional flow displacement leading to an additional flow velocity around the fuselage and the inboard wing compared to a configuration without a peniche. The strength of the peniche displacement effect is directly linked to the angle of attack of the configuration by means of a lift rise with increasing peniche height growing with increasing angle of attack. The peniche influence could be observed at every angle of attack, because also in the case of zero angle of attack an additional displacement takes place due to the peniche influence. The variation of the peniche height only changes the strength of the displacement effect, but it cannot be avoided in any case. 
- Regarding the improvement of existing half model wind tunnel correction methods no clear recommendations can be made for the use of one particular peniche height to suit all cryogenic high-lift testing at different Reynolds numbers. Unfortunately - no peniche can match the free flight result because of the local change of the flow on the inboard section. The appearance that the lift curve for a correction of a "special" peniche height matches well the free flight curve is coincidence, were a sum of modeling errors cancelling out each other. This was found e.g. for the DLR F11 $45 \mathrm{~mm}$ peniche configuration. Considering certain half model configurations it might only be possible to estimate from the experiments a peniche configuration with lowest interference effects at a specified Reynolds number.

It can be stated that the further development and application of powerful CFD methods in this context is more meaningful to improve significantly the prediction of wind tunnel results to free flight. It might only be possible by carefully performed analyses to answer the question after a half model-peniche configuration with lowest interference influences on the model flow.

Summarizing, the results presented in this paper show the huge potential of the application of modern CFD tools for the improvement of the wind tunnel technique in general especially for high Reynolds number testing in the ETW. The further development of the so-called "Numerical Wind Tunnel" is a promising way to come to better wind correction methods as well as to more accurate free flight predictions.

\section{References}

\footnotetext{
${ }^{1}$ Wichmann, G.: The objectives of DLR's ForMEx Project. European Wind tunnel Association (EWA), Initial Joint Workshop, ONERA Toulouse, October 18-20, 2004.

${ }^{2}$ Wichmann, G.: CFD Supported Wind Tunnel Testing - First Results of DLR Project ForMEx .EWA, European Windtunnel Association, 2nd Joint Workshop, Farnborough, March 20 - 21, 2006.

${ }^{3}$ Melber-Wilkending, S.; Wichmann, G.: Project ForMEx - A new CFD approach for transposition of Wind Tunnel data towards Flight Conditions. 15th AG STAB/DGLR Symposium Darmstadt, 29.11 - 1.12.2006.
}

${ }^{4}$ Melber-Wilkending, S.; Heidebrecht, A.; Wichmann, G.: A new approach in CFD supported Wind Tunnel testing. 25th International Congress of the Aeronautical Sciences, ICAS, Ham-burg, 3.-8. September, 2006.

${ }^{5}$ Melber-Wilkending, S.; Heidebrecht, A.; Wichmann, G.: CFD Validation by Wind Tunnel Measurements: Uncertainty Assessment by Numerical Simulation of Complete Wind Tunnel Flows. NATO-RTO, Applied Vehicle Technology, AVT 147, Symposium on "Computational Uncertainty in Military Vehicle Design", Greece, December 2007.

${ }^{6}$ Kroll, N.; Rossow, C.-C.; Becker, K.; Thiele, F.: MEGAFLOW - A Numerical Flow Simulation System. 21st ICAS congress, 1998, Melbourne, 13.09-18.09.1998, ICAS-98-2.7.4, 1998.

${ }^{7}$ Melber, S.; Heinrich, R.: Implementierung einer Praekonditionierungstechnik für Strömungen kleiner Mach-Zahlen im DLR TAU-Code und Anwendung im Hochauftrieb. 13th AG STAB/DGLR Symposium München, 12.-14. November 2002.

\footnotetext{
${ }^{8}$ Menter, F.R.: Two -- Equation Eddy -- Viscosity Turbulence Models for Engineering Applications. Journal of Aircraft, Vol. 32, No. 8, pp. 1598--1605, 1994.

${ }^{9}$ Dwight, R.: Investigation of the Implicit LU-SGS Scheme. DLR IB, 2005.

${ }^{10}$ Stahl, B.; Zhai, J.: Korrektur der im Rahmen der HAK1-Meßkampagne im Kryo-Kanal Köln gemessenen Polaren Modifikation für das KH3Y (F11) Modell, Confidential report, E9041 TR 084, ETW.
} 